Sharif University of Technology
Scientia Iranica
SCIENTIA

\title{
A modified model for stability analysis of narrow-width NEMS tweezers: Corrections due to surface layer, scale dependency, and force distributions
}

\author{
A. Koochi ${ }^{a, *}$, H. Hosseini-Toudeshky ${ }^{\mathrm{a}}$ and M. Abadyan ${ }^{\mathrm{b}}$ \\ a. Department of Aerospace Engineering, Amirkabir University of Technology, 424 Hafez Ave, Tehran, Iran. \\ b. Mechanical Engineering Group, Shahrekord Branch, Islamic Azad University, Shahrekord, Iran. \\ Received 10 September 2015; received in revised form 31 December 2015; accepted 26 April 2016
}

\author{
KEYWORDS \\ Nanotweezers; \\ Stability analysis; \\ Scale dependency; \\ Surface layer; \\ Strain gradient theory.
}

\begin{abstract}
Stability analysis and modeling the electromechanical response of nanotweezers are crucial for reliable designing and manufacturing of these nano-devices. Herein, a modified model is developed for static and dynamic stability analyses of nanotweezers with low width to thickness ration (narrow width). The surface elasticity was employed in conjunction with the strain gradient theory to consider the coupled effects of scale dependency, i.e. size-dependency of material characteristics and surface layer. The nonlinear governing equation was solved using analytical Rayleigh-Ritz Method (RRM). The influence of various parameters is addressed including scale dependency, surface stresses, damping parameter, and dispersion forces on the stability of the tweezers. Furthermore, the maximum length and the minimum gap of the tweezers were computed, which are important design parameters.
\end{abstract}

(C) 2017 Sharif University of Technology. All rights reserved.

\section{Introduction}

Manipulating nano-scale objects is of the great interest in nanotechnology and nanofabrication. Nanotweezers are basic manipulators, which are composed of two parallel arms with a distance in between [1-5]. By imposing a DC voltage differential between the arms, they move closer together; hence, the tweezers can manipulate ultra-small particles. At a critical voltage, i.e. the pull-in voltage, the Coulomb attraction overcomes the elastic resistance and the arms suddenly stick together. This critical voltage limits the range of stable displacement of the arms (tweezing range), and consequently, the size of objects that can bemanipulated by the tweezers. Therefore, predicting the stable tweezing range and the instability param-

\footnotetext{
*. Corresponding author. Tel.: +982164543224;

Fax: +982166959020

E-mail address: Ali.Koochi@aut.ac.ir (A. Koochi)
}

eters is crucial to design reliable nano-devices. As the dimensions of electromechanical systems reduce, dispersion forces, i.e. Casimir and van der Waals (vdW) attractions, appear [6-8]. At separations typically less than several micrometers, the dispersion forces between interacting surfaces can be described by the well-known Casimir force [6]. However, when the retardation is not significant, i.e. the separation is less than several ten nanometers, the quantum interactions should be modeled as vdW attraction $[2,7,8]$.

It should be noted that the nano-scale structural effects, i.e. size dependency and surface layer are the other crucial issues that should be considered in nanotweezers modeling. Some experimental works [911] demonstrate that the size dependency is an inherent property of conductive metals when the characteristic size of the structures is comparable with the internal material length scale. The length-scale parameter of materials can be measured experimentally via bending, torsion, or hardness tests [12,13]. Also, molecular 
dynamic simulations could be used to compute the material length-scale parameters [14]. The classical continuum model is not able to model the sizedependent behavior of materials and structures at submicron distances. In this regard, the non-classical theories, such as non-local elasticity [15], couple stress theory [16], strain gradient theory [10], modified couple stress theory [17], etc., have been developed to consider the effect of size on theoretical continuum models. One of the most successful size-dependent theories is the strain gradient elasticity theory proposed by Lam et al. [10]. This non-classic continuum theory introduces three material length-scale parameters to characterize the dilatation gradient tensor, the deviatoric stretch gradient tensor, and the symmetric rotation gradient tensor. The strain gradient theory has been applied to analyze mechanical behavior of ultra-small beams and other structures by many authors [18-21].

Beside the size dependency, the surface layer characteristics, i.e. the surface residual stress and surface stiffness, might be important in the behavior of nanoscale systems. Gurtin and Murdoch [22,23] developed a continuum theory for modeling both residual surface stress and surface elasticity. Wang et al. [24] studied the influences of surface tension and the residual stress on the elastic properties of nano structures. In recent years, some researchers have investigated the influence of surface layer on the pull-in characteristics of electromechanical systems including double-clamped nanobridge [25], cantilever nano-switches [26], graphite NEMS [27], and micro-plates [28].

In this work, the coupled effects of surface layer and size effect on the static and dynamic pull-in behaviors of nanotweezers have been investigated. The modified force distribution (i.e., electrical force, Casimir interaction, and van der Waals force) has been considered in the continuum model. Analytical Rayleigh-Ritz Method (RRM) is employed to solve the nonlinear governing equation of the system.

\section{Theory}

\subsection{Size effect}

Figure 1 shows the schematic representation of nanotweezers. Each arm of nanotweezers can be considered
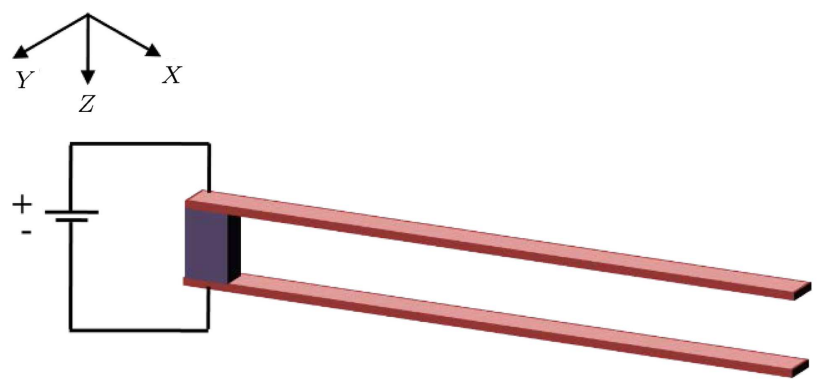

Figure 1. Schematic representation of nanotweezers. as a cantilever beam of length, $L$, a uniform crosssection of thickness, $h$, and width, $b$.

Regarding the strain gradient theory modified and suggested by Lam et al. [10], stored strain energy density in the linear elastic and isotropic material with small deformation is written as follows:

$$
\bar{U}=\frac{1}{2}\left(\sigma_{i j} \varepsilon_{i j}+p_{i} \gamma_{i}+\tau_{i j k}^{(1)} \eta_{i j k}^{(1)}+m_{i j}^{s} \chi_{i j}^{s}\right),
$$

where the components of Eq. (1) are explained in Appendix A.

For an Euler-Bernoulli beam, the displacement field can be expressed as [29]:

$$
u_{X}=-Z \frac{\partial W(X, t)}{\partial X}, \quad u_{Y}=0, \quad u_{Z}=W(X, t),
$$

where $W$ is the centerline deflection of the beam in the $Z$ direction; $u_{X}, u_{Y}$, and $u_{Z}$ are the displacement of the beam in the $X, Y$, and $Z$ directions, respectively.

Substituting Relations (A.9) into Eq. (1) after some elaboration and integrating over the beam volume, the bending strain energy is obtained as follows:

$$
\begin{aligned}
U_{B}= & \int_{V} \bar{U} d V=\frac{1}{2} \int_{0}^{L} \int_{A} E Z^{2}\left(\frac{\partial^{2} W}{\partial X^{2}}\right)^{2} \\
& +2 \mu l_{0}^{2} Z^{2}\left(\frac{\partial^{3} W}{\partial X^{3}}\right)^{2}+2 \mu l_{0}^{2} Z^{2}\left(\frac{\partial^{2} W}{\partial X^{2}}\right)^{2} \\
& +2 \mu l_{2}^{2} Z^{2}\left(\frac{\partial^{2} W}{\partial X^{2}}\right)^{2}+\frac{4}{25} \mu l_{1}^{2} Z^{2}\left(\frac{\partial^{3} W}{\partial X^{3}}\right)^{2} \\
& +\frac{48}{225} \mu l_{1}^{2}\left(\frac{\partial^{2} W}{\partial X^{2}}\right)^{2}+\frac{6}{25} \mu l_{1}^{2} Z^{2}\left(\frac{\partial^{3} W}{\partial X^{3}}\right)^{2} \\
& \left.+\frac{1}{75} \mu l_{1}^{2}\left(\frac{\partial^{2} W}{\partial X^{2}}\right)^{2}+\frac{1}{25} \mu l_{1}^{2}\left(\frac{\partial^{2} W}{\partial X^{2}}\right)^{2}\right\} d A d X \\
= & \frac{1}{2} \int_{0}^{L}\left[\left(E I+2 \mu A l_{0}^{2}+\frac{8}{15} \mu A l_{1}^{2}+\mu A l_{2}^{2}\right)\left(\frac{\partial^{2} W}{\partial X^{2}}\right)^{2}\right. \\
& \left.+A\left(2 \mu l_{0}^{2}+\frac{4}{5} \mu l_{1}^{2}\right)\left(\frac{\partial^{3} W}{\partial X^{3}}\right)^{2}\right] d X .
\end{aligned}
$$

In the above equation, $I$ is the second cross-section moment around $Y$ axis, and $A$ is the cross-section area.

\subsection{Strain energy in the surface layer}

According to surface elasticity theory, the strain energy in the surface layer $\left(U_{S}\right)$ is written as:

$$
U_{S}=\frac{1}{2} \int_{0}^{L} \oint_{\partial A} \tau_{i j} \varepsilon_{i j} d s d X,
$$


where the components of Eq. (4) are explained in Appendix B.

By substituting Eqs. (A.9a) and (B.3) in Eq. (4), the surface energy concludes as:

$$
\begin{aligned}
U_{S}= & \frac{1}{2} \int_{0}^{L} \oint_{\partial A}\left(-\left(\tau_{0}+E_{0} \frac{\partial u_{X}}{\partial X}\right) Z \frac{\partial^{2} W}{\partial X^{2}}\right. \\
& \left.+\tau_{0} n_{Z}^{2}\left(\frac{\partial W}{\partial X}\right)^{2}\right) d s d X=\frac{1}{2} \int_{0}^{L} \oint_{\partial A}\left(-\left(\tau_{0}\right.\right. \\
& \left.\left.+E_{0} Z \frac{\partial^{2} W}{\partial X^{2}}\right) Z \frac{\partial^{2} W}{\partial X^{2}}+\tau_{0} n_{Z}^{2}\left(\frac{\partial W}{\partial X}\right)^{2}\right) d s d X
\end{aligned}
$$

\subsection{Work of external forces}

Considering the distribution of external forces per unit length of the beam ( $\left.F_{\text {ext }}\right)$, the work done by these external forces can be obtained as:

$$
W_{\mathrm{ext}}=\int_{0}^{L} \int_{0}^{W} F_{\mathrm{ext}}(X, t) d W d X .
$$

The external force is the summation of electrical and dispersion forces (Casimir, or vdW, depends on the separation regime).

\subsubsection{Electrical force}

Batra et al. [30] showed that for two parallel narrow micro-beams, the electrical force can be obtained as:

$$
\begin{aligned}
f_{\text {elec }}= & \varepsilon_{0} \varepsilon_{r} \frac{b V^{2}}{g^{2}}\left[1+0.265\left(\frac{g}{b}\right)^{0.75}\right. \\
& \left.+0.53 \frac{h}{b}\left(\frac{g}{h}\right)^{0.5}\right]
\end{aligned}
$$

where $\varepsilon_{0}=8.854 \times 10^{-12} \mathrm{c}^{2} \mathrm{~N}^{-1} \mathrm{~m}^{-2}$ is the permittivity of vacuum, and $\varepsilon_{r}$ is the relative permittivity of dielectric.

It should be noted that by applying the external voltage to the nanotweezers, the arms will deflect to each other to reduce their gaps between $g$ and $g-W 1-W 2$. If both arms have the same geometry and material properties, their deflections will be equal $\left(W_{1}=W_{2}=W\right)$ [31]. Thus, by replacing $g$ with $g-2 W$, the electrostatic force per unit length of the arm is written as:

$$
\begin{aligned}
f_{\text {elec }}= & \varepsilon_{0} \varepsilon_{r} \frac{b V^{2}}{(g-2 W)^{2}}\left[1+0.265\left(\frac{g-2 W}{b}\right)^{0.75}\right. \\
& \left.+0.53 \frac{h}{b}\left(\frac{g-2 W}{h}\right)^{0.5}\right]
\end{aligned}
$$

\subsubsection{Casimir force}

By considering the finite dimension and edge effects, the Casimir force per unit length between two finite parallel plates can be explained as [32]:

$$
f_{\text {Cas }}=\frac{\pi^{2} \hbar c b}{240 g^{4}}+0.00231 \frac{\hbar c}{g^{3}}
$$

where $h=1.055 \times 10^{-34}$ Js is Planck's constant divided by $2 \pi$, and $c=2.998 \times 10^{8} \mathrm{~m} / \mathrm{s}$ is the light speed. Now, by replacing g with $g-2 W$, the modified Casimir force per unit length of beam is obtained as:

$$
\begin{aligned}
f_{\text {Cas }}= & \frac{\pi^{2} \hbar c b}{240(g-2 W)^{4}} \\
& +0.00231 \frac{\hbar c}{(g-2 W)^{3}} .
\end{aligned}
$$

\subsection{3. vdW force}

A reliable continuum model has been established to compute the vdW energy by double-volume integral of Lennard-Jones potential [33]. Using this approach, the vdW energy of two parallel beams with the same geometry and material properties can be defined as:

$$
f_{\mathrm{vdW}}=\frac{\bar{A} b}{6 \pi}\left[\frac{1}{g^{3}}+\frac{1}{(g+2 h)^{3}}-\frac{2}{(g+h)^{3}}\right],
$$

where $\bar{A}$ is the Hamaker constant.

By replacing $g$ with $g-2 W$, the vdW force per unit length can be obtained as:

$$
\begin{aligned}
f_{\mathrm{vdW}}= & \frac{\bar{A} b}{6 \pi}\left[\frac{1}{(g-2 W)^{3}}+\frac{1}{(g+2 h-2 W)^{3}}\right. \\
& \left.-\frac{2}{(g+h-2 W)^{3}}\right] .
\end{aligned}
$$

\subsection{Kinetic energy and damping loss}

The kinetic energy of each arm can be expressed as:

$$
T=\frac{1}{2} \int_{0}^{L} \int_{A} \rho\left[\left(\frac{\partial W}{\partial t}\right)^{2}\right] d A d X .
$$

Finally, the virtual work performed by damping effects of $W_{d}$ can be expressed as:

$$
W_{d}=\int_{0}^{L} \int_{0}^{W} c_{d} W_{t} d W d X
$$

where $c_{d}$ is the damping coefficient.

\subsection{Dimensionless energy of system}

The total energy of system can be summarized as: 


$$
\begin{aligned}
\Pi= & \frac{1}{2} \int_{0}^{L} \int_{A} \rho\left(\frac{\partial W}{\partial t}\right)^{2} d A d X-\int_{0}^{L} \int_{0}^{W} c_{d} \frac{\partial W}{\partial t} d W d X \\
& -\frac{1}{2} \int_{0}^{L} \oint_{\partial A}\left(-\left(\tau_{0}+E_{0} Z \frac{\partial^{2} W}{\partial X^{2}}\right) Z \frac{\partial^{2} W}{\partial X^{2}}\right. \\
& \left.+\tau_{0} n_{Z}^{2}\left(\frac{\partial W}{\partial X}\right)^{2}\right) d s d X-\frac{1}{2} \int_{0}^{L}\left[\left(E I+2 \mu A l_{0}^{2}\right.\right. \\
& \left.+\frac{8}{15} \mu A l_{1}^{2}+\mu A l_{2}^{2}\right)\left(\frac{\partial^{2} W}{\partial X^{2}}\right)^{2} \\
& \left.+I\left(2 \mu l_{0}^{2}+\frac{4}{5} \mu l_{1}^{2}\right)\left(\frac{\partial^{3} W}{\partial X^{3}}\right)^{2}\right] d X \\
& -\frac{1}{2} \int_{0}^{L}\left(\frac{E A}{2 L} \int_{0}^{L}\left(\frac{\partial W}{\partial X}\right)^{2} d X+N_{i}\right)\left(\frac{\partial W}{\partial X}\right)^{2} d X \\
& +\int_{0}^{L} \int_{0}^{w} F_{\text {ext }}(X, t) d w d X .
\end{aligned}
$$

Now, by substituting Eqs. (8), (10), and (12) into Eq. (15), considering $x=X / L$ and $w=W / g$, and some mathematical elaboration, the dimensionless total energy can be explained as:

$$
\begin{aligned}
\bar{\Pi}= & \frac{1}{2} \int_{0}^{1} \rho A\left(\frac{\partial w}{\partial \tau}\right)^{2} d X-\int_{0}^{1} \int_{0}^{w} \frac{\partial w}{\partial \tau} d w d x-\frac{1}{2} \int_{0}^{1} t_{0}\left(\frac{\partial w}{\partial x}\right)^{2} d x \\
& -\frac{1}{2} \int_{0}^{1}\left[f_{i}+6\left(\frac{\gamma}{\eta}\right)^{2} \int_{0}^{1}\left(\frac{\partial w}{\partial x}\right)^{2} d x\right]\left(\frac{\partial w}{\partial x}\right)^{2} d x \\
& -\frac{1}{2} \int_{0}^{1}\left[\left(1+\frac{\mu_{s}}{15}\left(30\left(\frac{l_{0}}{l_{2}}\right)^{2}+8\left(\frac{l_{1}}{l_{2}}\right)^{2}+15\right.\right.\right. \\
& \left.\left.+e_{0}\right)\right)\left(\frac{\partial^{2} w}{\partial x^{2}}\right)^{2}+\frac{\mu_{s}}{30(L / h)^{2}}\left(5\left(\frac{l_{0}}{l_{2}}\right)^{2}\right. \\
& \left.\left.+2\left(\frac{l_{1}}{l_{2}}\right)^{2}\right)\left(\frac{\partial^{3} w}{\partial x^{3}}\right)^{2}\right] d x+\int_{0}^{1} \int_{0}^{w}\left(\frac{2 \alpha^{2}}{(1-2 w)^{2}}[1\right. \\
& +0.265 \gamma^{0.75(1-2 w)^{0.75}} \\
& +0.53 \sqrt{\gamma \eta(1-2 w)}]) d w d
\end{aligned}
$$

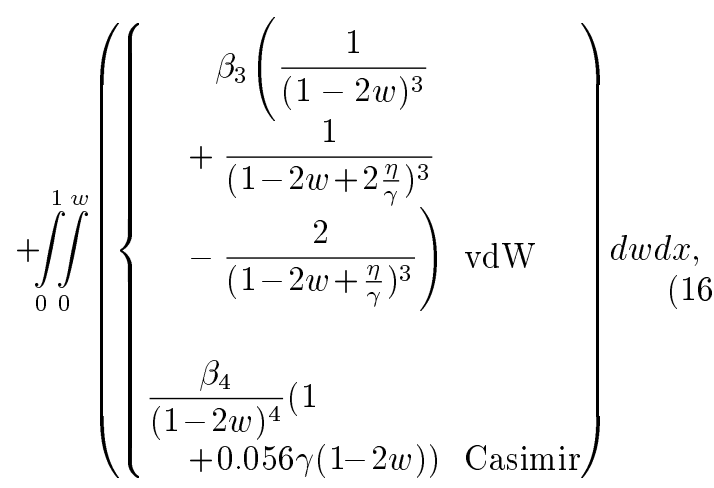

where the dimensionless parameters are identified as:

$$
\begin{aligned}
& \alpha^{2}=\frac{\varepsilon_{0} \varepsilon_{r} b V^{2} L^{4}}{2 g^{3} E I}, \\
& \beta_{n}=\left\{\begin{array}{l}
\frac{\bar{A} b L^{5}}{6 g^{4} \pi I} \quad \text { vdW interaction }(n=3) \\
\frac{\pi^{2} \hbar c b L^{4}}{240 g^{5} E I} \quad \text { Casimir interaction }(n=4)
\end{array}\right. \\
& \tau=\sqrt{\frac{E I}{\rho b h L^{4}}} t, \\
& e_{0}=\frac{E_{0} I_{0}}{E I}, \\
& t_{0}=\frac{S_{0} \tau_{0} L^{2}}{E I}, \\
& \mu_{s}=\frac{12 \mu}{E\left(h / l_{2}\right)^{2}}, \\
& \hat{c}=c_{d} L^{2} \sqrt{\frac{1}{\rho b h E I}}, \\
& \gamma=\frac{g}{b}, \\
& \eta=\frac{h}{b} .
\end{aligned}
$$

It should be noted that for rectangular section, we have:

$$
\begin{aligned}
& I_{0}=\int_{S} z^{2} d s=\frac{1}{2} b h^{2}+\frac{1}{6} h^{3}, \\
& S_{0}=\int_{S} n_{z}^{2} d s=2 b .
\end{aligned}
$$

\section{Solution methods}

\subsection{Rayleigh-Ritz Method (RRM)}

It is extremely difficult to calculate the exact mode shapes of the nano-beam based on strain gradient theory incorporated with the surface energy. Therefore, the well-known Galerkin method might not be easily applied to solve the governing equation. To overcome this difficulty, in this section, the Rayleigh-Ritz method 
is employed in conjunction with the total energy of the system (Eq. (16)) to solve the governing equation of the system. The displacement is expressed as a combination of a complete set of independent basis functions $\phi_{i}(x)$ in the form of:

$$
\hat{w}(x)=\sum_{i=1}^{n} q_{i}(t) \phi_{i}(x),
$$

where the index $i$ refers to the number of modes included in the simulation. We use the linear mode shapes of the nano-beam (based on the classic continuum theory) as basic functions in the Rayleigh-Ritz procedure:

$$
\begin{aligned}
& \phi_{i}(\xi)=\cosh \left(\omega_{i} \xi\right)-\cos \left(\omega_{i} \xi\right) \\
& -\frac{\cosh \left(\omega_{i}\right)+\cos \left(\omega_{i}\right)}{\sinh \left(\omega_{i}\right)+\sin \left(\omega_{i}\right)}\left(\sinh \left(\omega_{i} \xi\right)-\sin \left(\omega_{i} \xi\right)\right),
\end{aligned}
$$

where $\omega_{i}$ is the $i$ th root of characteristic equation of the cantilever beams in the classical theory. To minimize the total energy of the system, we must have:

$$
\frac{\partial \bar{\Pi}}{\partial q_{i}}=0 \quad i=0,1, \cdots, N .
$$

This leads to a system of time-dependent equations which can be solved numerically. Substituting Eqs. (16) and (19) into Eq. (21), assuming the orthogonality of $\phi_{i}(x)$, and then following some straightforward mathematical operations, a system of governing equations can be defined by Eq. (22) as shown in Box I.

In the equations shown in Box I, $N$ is the number of the considered terms of Rayleigh-Ritz. The Maple software is employed to numerically solve the system of equations.

\section{Results and discussion}

In this section, the influence of size effect, surface energies, and dispersion forces on the static and dynamic pull-in behaviors of nano-switches is studied.

\subsection{Validation}

In order to validate the presented model, the nonlinear governing differential equation is solved neglecting the size and surface effects, and the obtained results are compared with those of Ramezani [4] who studied the pull-in behavior of nanotweezers by parallel plate assumption for external forces (i.e., electrical, vdW,

$$
\begin{array}{r}
\ddot{q}_{i}+\hat{c} \dot{q}_{i}+\left[1+\frac{\mu_{s}}{15}\left(30\left(\frac{l_{0}}{l_{2}}\right)^{2}+8\left(\frac{l_{1}}{l_{2}}\right)^{2}+15+e_{0}\right)\right] \omega_{i}^{4} q_{i}-\frac{\mu_{s}}{30(L / h)^{2}}\left[5\left(\frac{l_{0}}{l_{2}}\right)^{2}+2\left(\frac{l_{1}}{l_{2}}\right)^{2}\right] \int_{0}^{1} \phi_{i} \frac{\partial^{6}}{\partial x^{6}}\left(\sum_{j=1}^{N} q_{j} \phi_{j}\right) \\
\quad-\int_{0}^{1} \phi_{i}\left\{f_{i}+t_{0}+6\left(\frac{\gamma}{\eta}\right)^{2}\left[\int_{0}^{1}\left(\sum_{j=1}^{N} q_{j} \frac{d \phi_{j}}{d x}\right)^{2} d x\right] \sum_{j=1}^{N} q_{j} \frac{d^{2} \phi_{j}}{d x^{2}}\right\} d x-\int_{0}^{1} \bar{F}_{\text {ext }} \phi_{i} d x+\frac{\mu_{s}}{30(L / h)^{2}}\left(5\left(\frac{l_{0}}{l_{2}}\right)^{2} 2\left(\frac{l_{1}}{l_{2}}\right)^{2}\right) \\
\left.\frac{\partial^{3}}{\partial X^{3}}\left(\sum_{j=1}^{N} q_{j} \phi_{j}\right) \frac{d^{2} \phi_{i}}{d X^{2}}\right|_{x=1}-\left.\frac{\mu_{s}}{30(L / h)^{2}}\left(5\left(\frac{l_{0}}{l_{2}}\right)^{2}+2\left(\frac{l_{1}}{l_{2}}\right)^{2}\right) \frac{\partial^{3}}{\partial X^{3}}\left(\sum_{j=1}^{N} q_{j} \phi_{j}\right) \frac{d^{2} \phi_{i}}{d X^{2}}\right|_{x=0}=0 ; \quad i=1,2, . ., N,
\end{array}
$$

where:

$$
\begin{aligned}
\bar{F}_{\text {ext }}= & \frac{2 \alpha^{2}}{\left(1-2 \sum_{j=1}^{N} q_{j} \phi_{j}\right)^{2}}\left[1+0.265 \gamma^{0.75}\left(1-2 \sum_{j=1}^{N} q_{j} \phi_{j}\right)^{0.75}+0.53 \sqrt{\gamma \eta\left(1-2 \sum_{j=1}^{N} q_{j} \phi_{j}\right)}\right] \\
& + \begin{cases}\beta_{3}\left(\frac{1}{\left(1-2 \sum_{j=1}^{N} q_{j} \phi_{j}\right)^{3}}+\frac{1}{\left(1-2 \sum_{j=1}^{N} q_{j} \phi_{j}+2 \frac{\eta}{\gamma}\right)^{3}}-\frac{2}{\left(1-2 \sum_{j=1}^{N} q_{j} \phi_{j}+\frac{\eta}{\gamma}\right)^{3}}\right) & \mathrm{vdW} \\
\frac{\beta_{4}}{\left(1-2 \sum_{j=1}^{N} q_{j} \phi_{j}\right)^{4}}\left(1+0.056 \gamma\left(1-2 \sum_{j=1}^{N} q_{j} \phi_{j}\right)\right) & \text { Casimir }\end{cases}
\end{aligned}
$$


Table 1. A comparison between the pull-in voltages calculated by presented model and literature $(\gamma=1)$.

\begin{tabular}{|c|c|c|c|}
\hline & Analytical [4] & Numerical [4] & RRM \\
\hline $\begin{array}{c}\alpha_{P I}, \text { vdW regime } \\
\left(\beta_{3}=0.3\right)\end{array}$ & 0.3346 & 0.3346 & 0.338 \\
\hline $\begin{array}{c}\alpha_{P I}, \text { Casimir regime } \\
\left(\beta_{4}=0.2\right)\end{array}$ & 0.3728 & 0.3728 & 0.373 \\
\hline
\end{tabular}

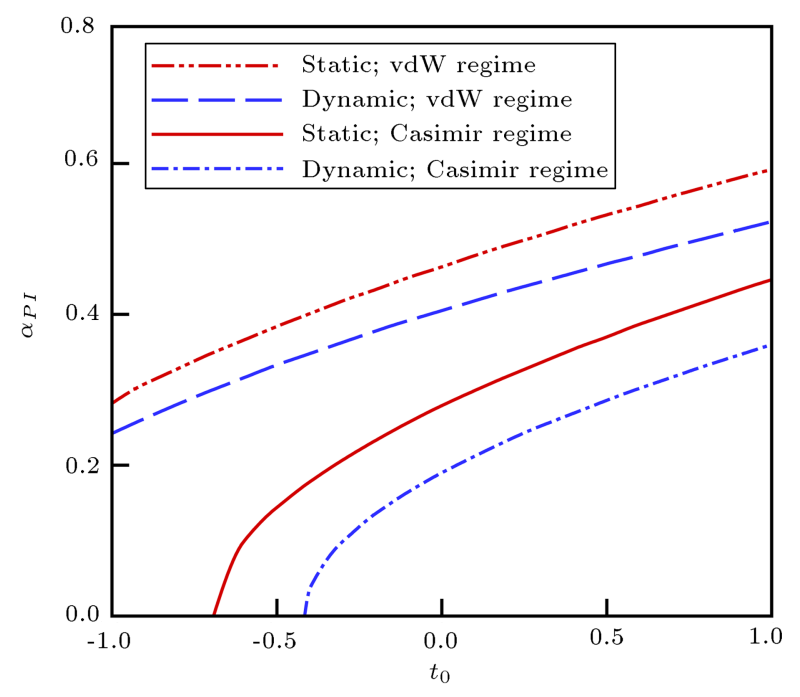

Figure 2. Influence of residual surface stress on the pull-in voltage $\left(\gamma=1, \eta=0.2, \beta_{n}=0.25\right)$.

and Casimir). The values of non-dimensional static pull-in voltage $\left(\alpha_{P I}\right)$ for the system, calculated by different methods, are presented in Table 1 . It is obvious from the reported results that the values of computed static pull-in voltage $\left(\alpha_{P I}\right)$ agrees well with those reported in the literature. On the other hand, Table 1 reveals that for wide beams, the results of the presented method converge with results obtained by the parallel plate assumption.

\subsection{Surface layer}

The influence of surface residual stress and dispersion forces on the static and dynamic pull-in voltages of nanotweezers is presented in Figure 2. This figure shows that by increasing the surface residual stress, the pull-in voltage enhances. It should be noted that $t_{0}=$ 0 is subjected to neglecting surface stress condition. Figure 2 reveals that surface stress induces hardening or softening effect depending on its sign; when the surface stress is positive, the surface effect increases the pull-in voltage; if the surface stress is negative, the surface effect reduces the pull-in voltage. Furthermore, Figure 2 shows that the dispersion forces decrease the pull-in voltage. The dynamic pull-in voltage of nanotweezers is lower than static pull-in voltage as the result of inertia force.

The influences of surface elastic modulus and dispersion forces on the static and dynamic pull-in voltages are presented in Figure 3. This figure reveals

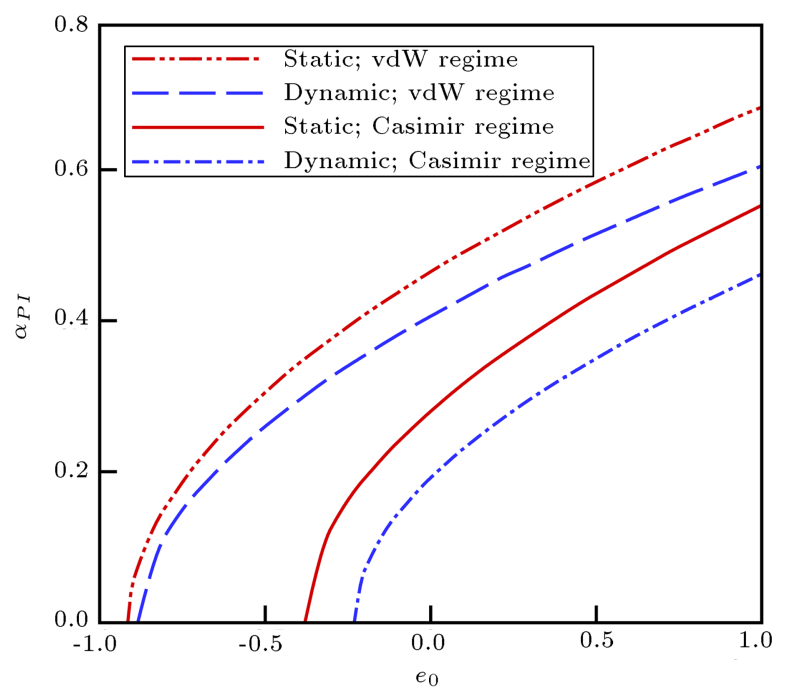

Figure 3. Influence of surface elasticity on the pull-in voltage $\left(\gamma=1, \eta=0.2, \beta_{n}=0.25\right)$.

that by increasing the surface elastic modulus, the pullin voltage enhances. It should be noted that $e_{0}=0$ is subjected to ignoring surface elastic modulus effect $\left(E_{0}=0\right)$. Figure 3 reveals that surface elastic modulus induces hardening or softening effect depending on the sign of $E_{0}$; when the surface elastic modulus is positive, the surface effect increases the pull-in voltage; if the surface elastic modulus is negative, the surface effect reduces the pull-in voltage.

\subsection{Size effect and surface effect}

The influences of size effect and surface residual stress on the static and dynamic pull-in voltages of nanotweezers are shown in Figure 4 for $l_{0}=l_{1}=l_{2}$. This figure reveals that decreasing $h / l_{2}$ results in decreasing the pull-in voltage. It should be noted that decrease in $h / l_{2}$ value corresponds to an increase in size effect. This means that size effect provides a hardening behavior that enhances the elastic resistance and consequent pull-in voltage of the nanotweezers.

\subsection{Free standing nanotweezers}

When the gap between the arms is sufficiently small, the arms of nanotweezers can adhere to each other due to the dispersion forces even without voltage difference. The critical values of vdW or Casimir forces, $\beta_{n C}$, and the corresponding critical value of arm tip deflection, $w_{C}$, can be acquired by setting $\alpha=0$, and then solving the governing equation. The maximum length 


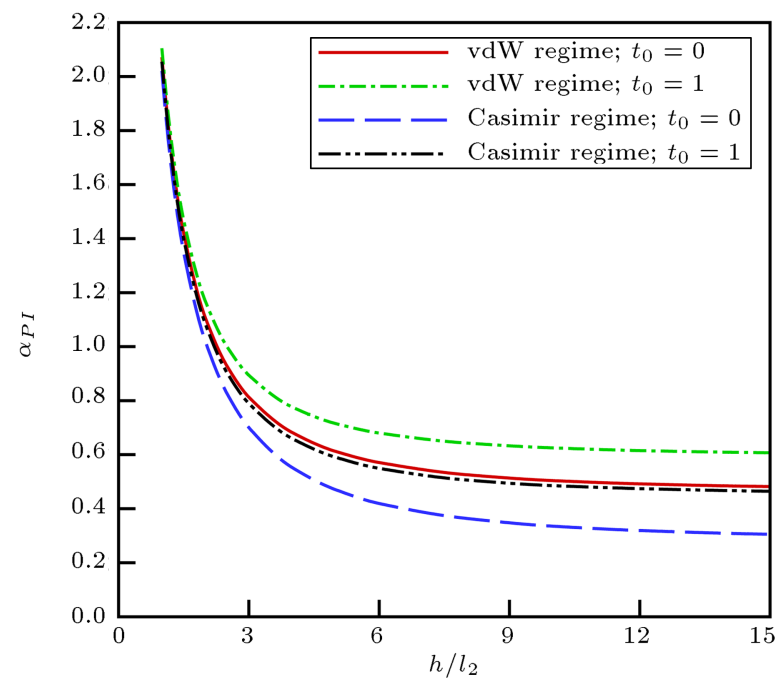

(a)

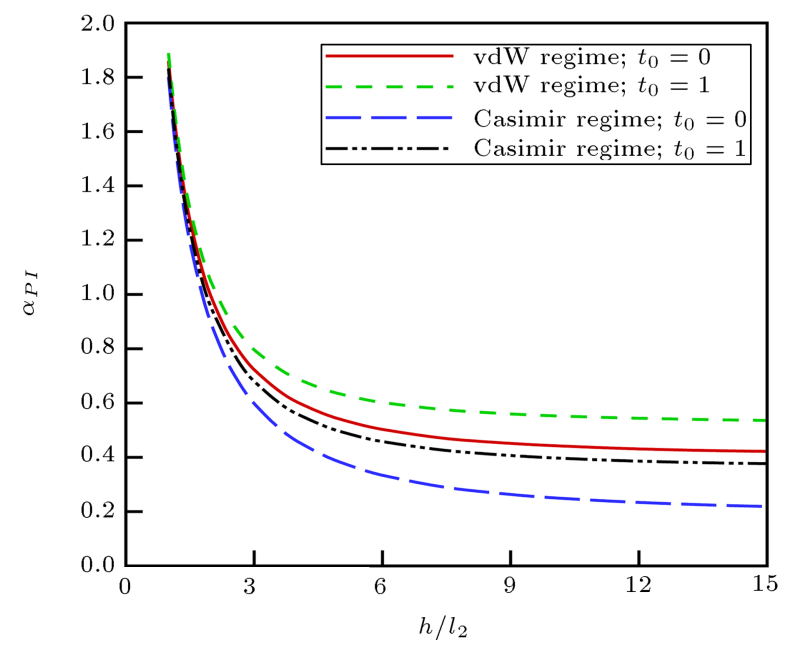

(b)

Figure 4. Influence of size effect and surface residual stress on the static pull-in voltage: (a) Static, and (b) dynamic.

of the arms, $L_{\max }$, in a way that the arms do not stick together without any voltage difference is called the detachment length [34]. The detachment length is the maximum permissible length of the freestanding arms. On the other hand, if the length of the arms are known, there is a minimum gap, $g_{\text {min }}$, which prevents stiction between the arms due to the dispersion forces. The maximum length and minimum gap are very important in designing nanotweezers. Substituting $\beta_{n C}$ into definition of $\beta_{n}$ in Eq. (17b), one can calculate the values of $L_{\max }$ and $g_{\min }$. For vdW regime, we have:

$$
\begin{aligned}
& L_{\max }=\sqrt[5]{\frac{6 g^{4} E I \beta_{3 C}}{\pi C_{6} \rho b}}, \\
& g_{\min }=\sqrt[4]{\frac{\pi C_{6} \rho b L^{5}}{6 E I \beta_{3 C}}},
\end{aligned}
$$

and for Casimir regime, one can obtain:

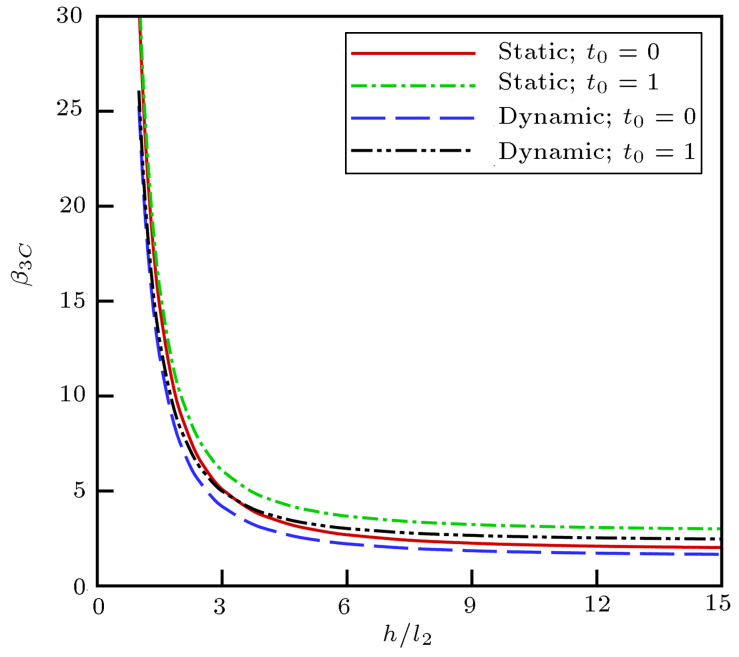

(a)

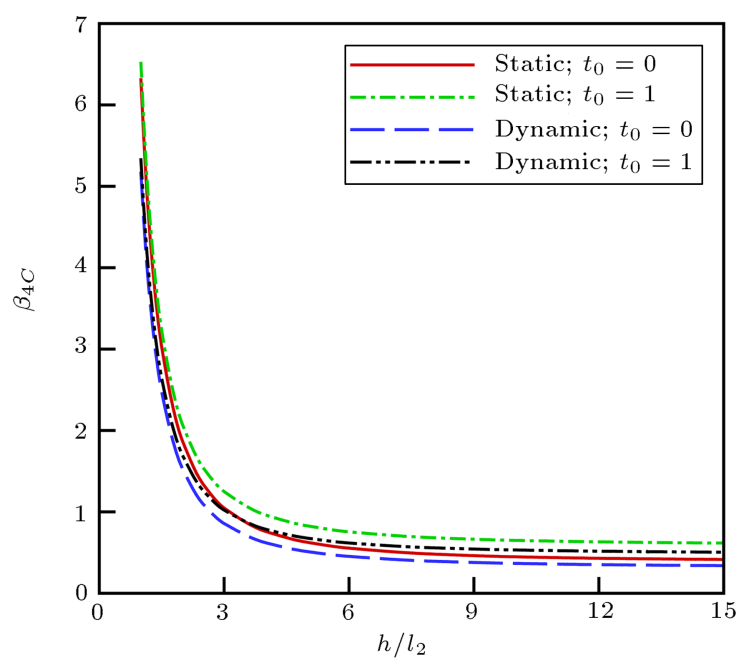

(b)

Figure 5. Influence of size effect and surface residual stress on the critical dispersion forces: (a) vdW, and (b) Casimir.

$$
\begin{aligned}
& L_{\max }=\sqrt[4]{\frac{240 g^{5} E I \beta_{4 C}}{\pi^{2} \hbar c b}}, \\
& g_{\min }=\sqrt[5]{\frac{\pi^{2} \hbar c b L^{4}}{240 E I \beta_{4 C}}}
\end{aligned}
$$

Figure 5 shows the critical values of dispersion forces $\left(\beta_{n C}\right)$ for different values of parameter $h / l_{2}$. This figure reveals that decreasing the parameter $h / l_{2}$ (i.e., increasing the size parameter or reducing the thickness) increases $\beta_{n C}$ value. Hence, the size parameter increases the detachment length and reduces the minimum gap (see Eqs. (24) and (25)). Furthermore, this figure shows that surface residual stress increases the critical values of dispersion forces $\left(\beta_{n C}\right)$.

\subsection{Effect of damping}

The influence of the damping on the dynamic behavior of vibrating nanotweezers is illustrated in Figure 6. The 


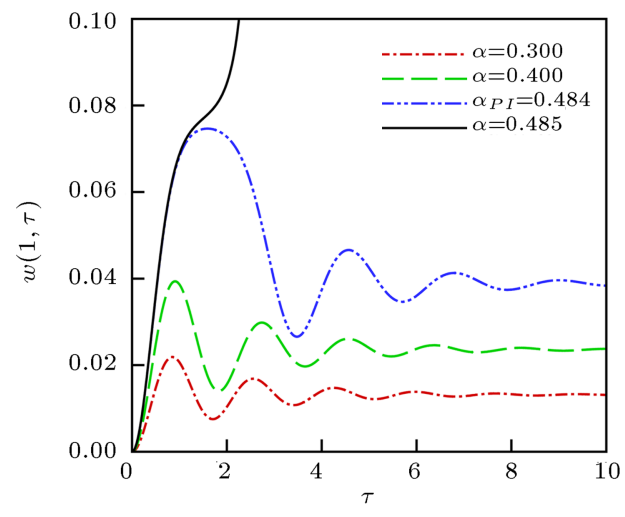

(a)

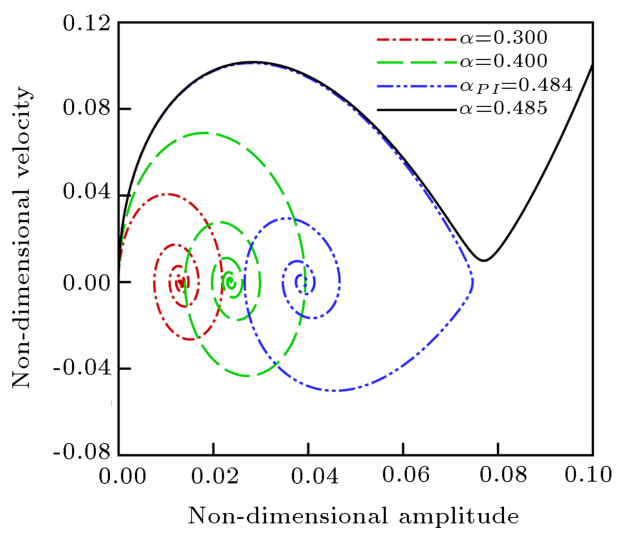

(c)

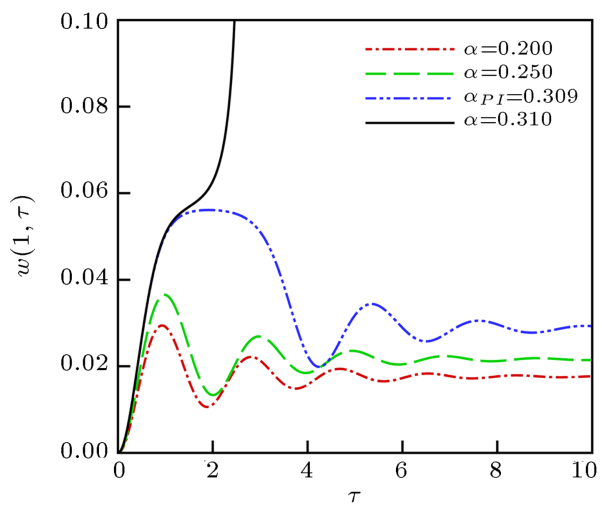

(b)

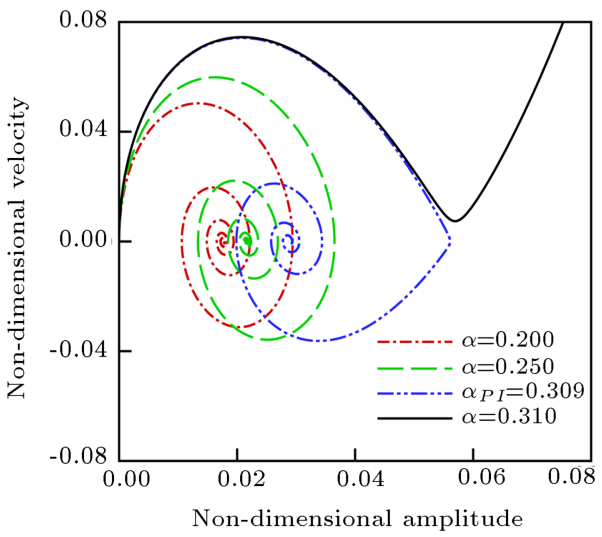

(d)

Figure 6. Influence of damping parameter on the dynamic behavior of nanotweezers $\left(h / l_{2}=10 ; t_{0}=0.1, e_{0}=0.05\right.$, $\beta_{n}=0.25, \hat{c}=1$ ): (a) Time history, vdW, (b) time history, Casimir, (c) phase plane, vdW, and (d) phase plane, Casimir.

obtained results show that the stable center equilibrium point becomes a stable focus point when the damping parameter is taken into account. It can be concluded that the nanotweezers make convergent oscillations near the focus point because of the damping and make periodic oscillations if the damping is neglected. On the other hand, the second equilibrium point is unstable saddle point for any value. When the actuation voltage reaches the pull-in voltage, the trajectories, which are attracted to the stable focus due to the damping effect, diverge and the nanotweezers become unstable.

\section{Conclusions}

In this work, a modified formulation was derived for modelling the pull-in behavior of nanotweezers. The surface elasticity in conjunction with the strain gradient theory was employed to consider the coupled effects of size dependency of material characteristics and surface layer. It was found that for positive surface stress, the effect of surface layer increased the pullin voltage. However, if the surface stress be negative, the surface effect reduces the pull-in voltage. On the other hand, the size phenomenon always increases the pull-in voltage of nanotweezers, while the dispersion forces decrease the instability voltage. The detachment length and minimum gap of the tweezers were determined. The dynamic pull-in voltage of nanotweezers was lower than static pull-in voltage as a result of inertia forces. When the damping effect was included in the simulations, the stable center point became the stable focus point.

\section{References}

1. Kim, P. and Lieber, C.M. "Nanotube nanotweezers", Science, 286(5447), pp. 2148-2150 (1999).

2. Wang, G.W., Zhang, Y., Zhao, Y.P. and Yang, G.T. "Pull-in instability study of carbon nanotube tweezers under the influence of van der Waals forces", $J$. Micromech. Microeng., 14, pp. 1119-1125 (2004).

3. Akita, S. and Nakayama, Y. "Manipulation of nanomaterial by carbon nanotube nanotweezers in scanning probe microscope", Jpn. J. App. Phys., 41(6S), p. 4242 (2002).

4. Ramezani, A. "Ability analysis of electrostatic nanotweezers", Physica E, 43(10), pp. 1783-1791 (2011).

5. Farrokhabadi, A., Koochi, A. and Abadyan, M. "Modeling the instability of CNT tweezers using a 
continuum model", Microsys. Tech., 20(2), pp. 291$302(2014)$.

6. Lin, W.H. and Zhao, Y.P. "Dynamics behavior of nanoscale electrostatic actuators", Chin. Phys. Lett., 20, pp. 2070-2073 (2003).

7. Lin, W.H. and Zhao, Y.P. "Casimir effect on the pull-in parameters of nanometer switches", Microsys.. Tech., 11, pp. 80-85 (2005).

8. Guo, J.G. and Zhao, Y.P. "Influence of van der Waals and Casimir forces on electrostatic torsional actuators", J. Microelectromech. Sys., 13(6) pp. 10271035 (2004).

9. Fleck, N.A., Muller, G.M., Ashby, M.F. and Hutchinson, J.W. "Strain gradient plasticity: theory and experiment", Acta Metallurgica et Materialia, 42(2), pp. 475-487 (1994).

10. Lam, D.C.C., Yang, F., Chong, A.C.M., Wang, J. and Tong, P. "Experiments and theory in strain gradient elasticity", J. Mech. Phys. Solids, 51(8), pp. 1477-1508 (2003).

11. McFarland, A.W. and Colton, J.S. "Thermoplastic polymer microcantilever sensors fabricated via micromolding", J. Micromech. Microeng., 15(1), pp. 10601067 (2005).

12. Nix, W.D. and Gao, H. "Indentation size effects in crystalline materials: A law for strain gradient plasticity", J. Mech. Phys. Solid, 46, pp. 411-425 (1998).

13. Cao, Y., Nankivil, D.D., Allameh, S. and Soboyejo W.O. "Mechanical properties of Au films on silicon substrates", Mater. Manufact. Processes, 22, pp. 187194 (2007).

14. Maranganti, R. and Sharma, P. "A novel atomistic approach to determine strain-gradient elasticity constants: tabulation and comparison for various metals, semiconductors, silica, polymers and the (Ir) relevance for nanotechnologies", J. Mech. Phys. Solid, 55(9) pp. 1823-1952 (2007).

15. Eringen, A.C. and Edelen, D.G.B. "On nonlocal elasticity", Int. J. Eng. Sci., 10(3), pp. 233-248 (1972).

16. Ejike, U.B. "The plane circular crack problem in the linearized couple-stress theory", Int. J. Eng. Sci., 7(9), pp. 947-961 (1969).

17. Yang, F., Chong, A.C.M., Lam, D.C.C. and Tong, P. "Couple stress based strain gradient theory for elasticity", Int. J. Solid Struct., 39(10), pp. 2731-2743 (2002).

18. Wang, B., Zhou, S., Zhao, J. and Chen, X. "Pullin instability of circular plate MEMS: A new model based on strain gradient elasticity theory", Int. J. App. Mech., 4(1), p. 1250003 (2012).

19. Ansari, R., Gholami, R., Mohammadi, V. and Shojaei, M.F. "Size dependent pull-In instability of hydrostatically and electrostatically actuated circular microplates", J. Comput. Nonlinear Dyn., 8(2), p. 021015 (2013).
20. Wang, B., Zhao, J. and Zhou, S. "A micro scale Timoshenko beam model based on strain gradient elasticity theory", Eur. J. Mech. A, 29(4), pp. 591-599 (2010).

21. Koochi, A., Sedighi, H.M. and Abadyan, M. "Modeling the size dependent pull-in instability of beam-type NEMS using strain gradient theory", Latin American J. Solid Strucs., 11, pp. 1806-1829 (2014).

22. Gurtin, M.E. and Murdoch, A.I. "A continuum theory of elastic material surfaces", Arch. Rational Mech. Anal., 57(4), pp. 291-323 (1975).

23. Gurtin, M.E. and Murdoch, A.I. "Surface stress in solids", Int. J. Solid Struct., 14(6), pp. 431-440 (1978).

24. Wang, Z.Q., Zhao, Y.P. and Huang, Z.P. "The effects of surface tension on the elastic properties of nano structures", Int. J. Eng. Sci., 48, pp. 140-150 (2010).

25. Fu, Y. and Zhang, J. "Size-dependent pull-in phenomena in electrically actuated nanobeams incorporating surface energies", App. Math. Model., 35(2), pp. 941951 (2011).

26. Koochi, A., Hosseini-Toudeshky, H., Ovesy, H.R. and Abadyan, M. "Modeling the influence of surface effect on instability of nano-cantilever in presence of van der Waals force", Int. J. Struct. Stab. Dyn., 13(04), p. 1250072 (2013).

27. Rokni, H. and Lu, W. "A continuum model for the static pull-in behavior of graphene nanoribbon electrostatic actuators with interlayer shear and surface energy effects", J. App. Phys., 113(15), p. 153512 (2013).

28. Ansari, R. and Sahmani, S. "Surface stress effects on the free vibration behavior of nanoplates", Int. J. Eng. Sci., 49(11), pp. 1204-1215 (2011).

29. Dym, C.L. and Shames, I.H., Solid Mechanics, New York, McGraw-Hill (1973).

30. Batra, R.C., Porfiri, M. and Spinello, D. "Electromechanical model of electrically actuated narrow microbeams", J. Microelectromech. Sys., 15(5), pp. 1175-1189 (2006).

31. Farrokhabadi, A., Koochi, A., Kazemi, A. and Abadyan, "Effect $\mathrm{s}$ of size-dependent elasticity on stability of nanotweezers", Appl. Math. Mech. -Engl. Ed., 35(12), pp. 1573-1590 (2014).

32. Gies, H. and Klingmüller, K. "Casimir edge effects", Physical Rev. Lett., 97(22), p. 220405 (2006).

33. Lennard-Jones, J.E. "Perturbation problems in quantum mechanics", Proceedings of the Royal Society of London. Series A, Containing Papers of a Mathematical and Physical Character, 129(811) pp. 598-615 (1930).

34. Lin, W.H. and Zhao, Y.P. "Nonlinear behavior for nanoscale electrostatic actuators with Casimir force", Chaos Solitons Fractals, 23(5), pp. 1777-1785 (2005). 


\section{Appendix A}

\section{Strain Gradient theory}

The components of strain energy in strain gradient theory are:

$$
\begin{aligned}
\varepsilon_{i j}= & \frac{1}{2}\left(u_{i, j}+u_{j, i}\right), \\
\gamma_{i}= & \varepsilon_{m m, i}, \\
\eta_{i j k}^{(1)}= & \frac{1}{3}\left(\varepsilon_{j k, i}+\varepsilon_{k i, j}+\varepsilon_{i j, k}\right) \\
& -\frac{1}{15} \delta_{i j}\left(\varepsilon_{m m, k}+2 \varepsilon_{m k, m}\right) \\
& -\frac{1}{15}\left[\delta_{j k}\left(\varepsilon_{m m, i}+2 \varepsilon_{m i, m}\right)\right. \\
& \left.+\delta_{k i}\left(\varepsilon_{m m, j}+2 \varepsilon_{m j, m}\right)\right], \\
\chi_{i j}^{s}= & \frac{1}{2} e_{j k l} u_{l, k i} .
\end{aligned}
$$

In the above equations, $u_{i}, \gamma_{i}, \eta_{i j k}^{(1)}, \chi_{i j}^{s}, \delta_{i j}$, and $e_{i j k}$ indicate displacement vector, dilatation gradient vector, deviatoric stretch gradient tensor, symmetric rotation gradient tensor, Kronocker delta, and permutation symbol, respectively. Also, $\varepsilon_{i j}, \sigma_{i j}, p_{i}, \tau_{i j k}^{(1)}$ and $m_{i j}^{s}$ are strain tensor, Cauchy's stress, and high order stress tensors, respectively, which are identified as follows [10]:

$$
\begin{aligned}
& \sigma_{i j}=2 \mu\left(\varepsilon_{i j}+\frac{\nu}{1-2 \nu} \varepsilon_{m m} \delta_{i j}\right), \\
& p_{i}=2 \mu l_{0}^{2} \gamma_{i}, \\
& \tau_{i j k}^{(1)}=2 \mu l_{1}^{2} \eta_{i j k}^{(1)}, \\
& m_{i j}^{s}=2 \mu l_{2}^{2} \chi_{i j}^{s} .
\end{aligned}
$$

In the above equations, $\nu$ and $\mu$ are Poisson's ratio and shear modulus, respectively. Also, $l_{0}, l_{1}$, and $l_{2}$ are additional material length-scale parameters which appear in the constitutive equations of higher order stresses.

Substituting the linear displacement field of Eq. (2) into Eqs. (A.1)-(A.8), the non-zero component is obtained as the following relations:

$$
\begin{aligned}
& \varepsilon_{11}=-Z \frac{\partial^{2} W}{\partial X^{2}}, \\
& \sigma_{11}=-E Z \frac{\partial^{2} W}{\partial X^{2}}, \\
& \gamma_{1}=-Z \frac{\partial^{3} W}{\partial X^{3}}, \quad \gamma_{3}=-\frac{\partial^{2} W}{\partial X^{2}},
\end{aligned}
$$

$$
\begin{aligned}
& p_{1}=-2 \mu l_{0}^{2} Z \frac{\partial^{3} W}{\partial X^{3}}, \quad p_{3}=-2 \mu l_{0}^{2} \frac{\partial^{2} W}{\partial X^{2}}, \quad \text { (A.9d) } \\
& \chi_{12}^{s}=\chi_{21}^{s}=-\frac{1}{2} \frac{\partial^{2} W}{\partial X^{2}}, \\
& m_{12}^{s}=m_{21}^{s}=-\mu l_{2}^{2} \frac{\partial^{2} W}{\partial X^{2}}, \\
& \eta_{111}^{(1)}=-\frac{2}{5} Z \frac{\partial^{3} W}{\partial X^{3}}, \\
& \eta_{113}^{(1)}=\eta_{131}^{(1)}=\eta_{311}^{(1)}=-\frac{4}{15} \frac{\partial^{2} W}{\partial X^{2}}, \\
& \eta_{122}^{(1)}=\eta_{212}^{(1)}=\eta_{221}^{(1)}=\eta_{133}^{(1)}=\eta_{313}^{(1)}=\eta_{331}^{(1)}=\frac{1}{5} Z \frac{\partial^{3} W}{\partial X^{3}}, \\
& \eta_{223}^{(1)}=\eta_{232}^{(1)}=\eta_{322}^{(1)}=\frac{1}{15} \frac{\partial^{2} W}{\partial X^{2}}, \\
& \eta_{333}^{(1)}=\frac{1}{5} \frac{\partial^{2} W}{\partial X^{2}}, \\
& \tau_{111}^{(1)}=-\frac{4}{5} \mu l_{1}^{2} Z \frac{\partial^{3} W}{\partial X^{3}}, \\
& \tau_{113}^{(1)}=\tau_{131}^{(1)}=\tau_{311}^{(1)}=-\frac{8}{15} \mu l_{1}^{2} \frac{\partial^{2} W}{\partial X^{2}}, \\
& \tau_{122}^{(1)}=\tau_{212}^{(1)}=\tau_{221}^{(1)}=\tau_{133}^{(1)}=\tau_{313}^{(1)}=\tau_{331}^{(1)}=\frac{\partial^{2} W}{\partial X^{2}} \mu \\
& \tau_{1}^{2} Z \frac{2}{15} \mu l_{1}^{2} \frac{\partial^{2} W}{\partial X^{2}},
\end{aligned}
$$

\section{Appendix B}

\section{Surface elasticity}

According to continuum theory proposed by Gurtin and Murdoch [22,23], the in-plane components of the surface stress tensor are given by:

$$
\begin{aligned}
\tau_{\alpha \beta}= & \mu_{0}\left(u_{\alpha, \beta}+u_{\beta, \alpha}\right)+\left(\lambda_{0}+\tau_{0}\right) u_{p, p} \delta_{\alpha \beta} \\
& +\tau_{0}\left(\delta_{\alpha \beta}-u_{\beta, \alpha}\right),
\end{aligned}
$$

where $\mu_{0}$ and $\lambda_{0}$ are the surface elastic constants, $\tau_{0}$ is the residual surface stress. The out-of-plane components of the surface stress tensor are given by [22]:

$$
\tau_{n \alpha}=\tau_{0}\left(u_{n, \alpha}\right) .
$$

By substituting Eq. (2) into Eqs. (B.1) and (B.2), the 
non-zero components of surface stress are obtained as:

$$
\tau_{X X}=\tau_{0}+E_{0} \frac{\partial u_{x}}{\partial X}, \quad \tau_{n X}=\tau_{0} \frac{\partial u_{n}}{\partial X},
$$

where $E_{0}=\lambda_{0}+2 \mu_{0}$ is the surface elastic modulus, which can be determined from atomistic calculations [22].

\section{Biographies}

Ali Koochi received the MSc degree in Aerospace Engineering from Sharif University of Technology, Tehran, Iran, and is currently a PhD student in Aerospace Engineering in Amirkabir University of Technology, Tehran, Iran. He is interested in modeling nano-structures and
NEMS using nano-scale continuum models.

Hossein Hosseini-Toudeshky obtained a PhD degree in Solid Mechanics, University of New South Wales, Sydney, Australia, 1996, and is currently a Professor of Aerospace Engineering in Amirkabir University of Technology, Tehran, Iran. His current research interests include the stability analysis and fatigue and fracture Mechanics.

Mohamadreza Abadyan received the MSc and PhD degrees in Aerospace Engineering from Sharif University of Technology, Iran, in 2004 and 2010, respectively. His current research interests include the pull-in performance of MEMS/NEMS and mechanical behavior of polymer/composites. 